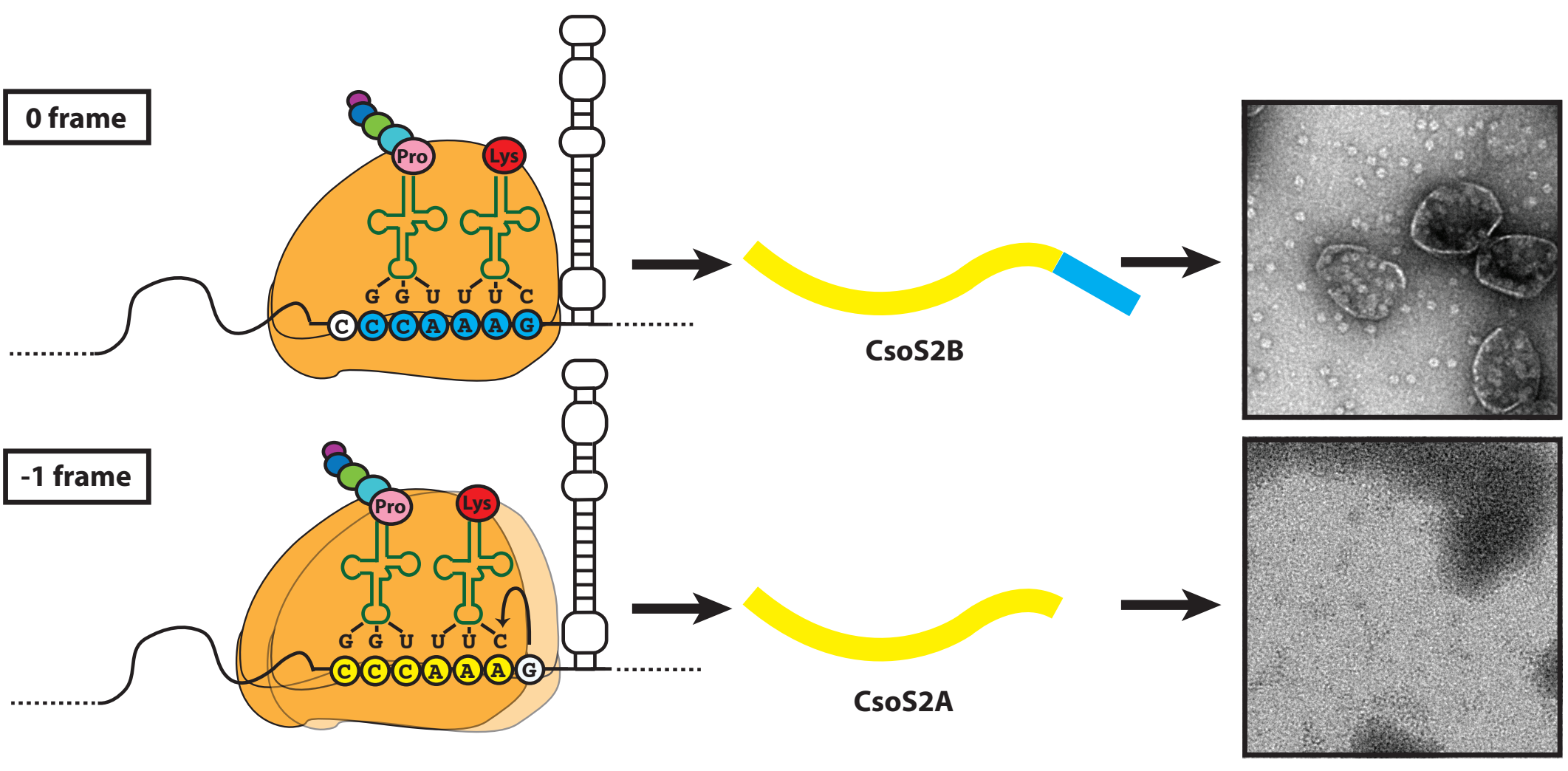




\section{Programmed ribosomal frameshifting mediates expression of the $\alpha$-carboxysome}

3 Thawatchai Chaijarasphong ${ }^{1}$, Robert J. Nichols ${ }^{2}$, Kaitlyn E. Kortright ${ }^{2}$, Charlotte F. Nixon ${ }^{2}$, Poh

4 K. Teng ${ }^{2}$, Luke M. Oltrogge ${ }^{2}$, and David F. Savage ${ }^{1,2,3, *}$

5

$6{ }^{1}$ Department of Chemistry, UC Berkeley, Berkeley, CA, 94720, USA

$7 \quad{ }^{2}$ Department of Molecular \& Cell Biology, UC Berkeley, Berkeley, CA, 94720, USA

$8{ }^{3}$ Energy Biosciences Institute, UC Berkeley, Berkeley, CA 94720, USA

9

$10 *$ To whom correspondence should be addressed. Email: savage@ berkeley.edu.

11

12 


\section{Abbreviations}

14 CCM, Carbon dioxide concentrating mechanism; PRF, programmed ribosomal frameshifting;

$15 \mathrm{CB}$, carboxysomes; BMC, bacterial microcompartment; $\mathrm{HnCB}, H$. neapolitanus carboxysomes;

16 RuBisCO, Ribulose-1,5- bisphosphate Carboxylase/ Oxygenase; MW, molecular weight; PAGE,

17 polyacrylamide gel electrophoresis; EM, electron microscopy; MS, mass spectrometry; MS/MS,

18 tandem mass spectrometry; MALDI-TOF, Matrix assisted laser desorption/ionization - time of

19 flight; GFP, Green fluorescent protein; RFP, Red fluorescent protein; FMDV, Foot-and-Mouth-

20 Disease Virus; IRES, Internal ribosome entry site

\section{Keywords}

22 Bacterial microcompartments; CsoS2; co-translational regulation; carboxysome 


\section{Abstract}

24 Many bacteria employ a protein organelle, the carboxysome, to catalyze carbon dioxide 25 fixation in the Calvin Cycle. Only ten genes from H. neapolitanus are sufficient for heterologous 26 expression of carboxysomes in E. coli, opening the door to detailed mechanistic analysis of the 27 assembly process of this 200+ MDa complex. One of these genes, csoS2, has been implicated in 28 assembly but ascribing a molecular function is confounded by the observation that the single $29 \operatorname{csoS} 2$ gene yields expression of two gene products and both display an apparent molecular weight 30 incongruent with the predicted amino acid sequence. Here, we elucidate the co-translational 31 mechanism responsible for the expression of the two protein isoforms. Specifically, csoS2 was 32 found to possess -1 frameshifting elements that lead to the production of the full-length protein, $33 \mathrm{CsoS} 2 \mathrm{~B}$, and a truncated protein, CsoS2A, which possesses a C-terminus translated from the 34 alternate frame. The frameshifting elements comprise both a ribosomal slippery sequence and 3', 35 secondary structure, and ablation of either sequence is sufficient to eliminate the slip. Using these 36 mutants, we investigated the individual roles of $\mathrm{CsoS} 2 \mathrm{~B}$ and $\mathrm{CsoS} 2 \mathrm{~A}$ on carboxysome formation.

37 In this in vivo formation assay, cells expressing only the CsoS2B isoform were capable of 38 producing intact carboxysomes, while those with only CsoS2A were not. Thus, we have answered 39 a long-standing question about the nature of $\mathrm{CsoS} 2$ in this model microcompartment and 40 demonstrate that $\mathrm{CsoS} 2 \mathrm{~B}$ is functionally distinct from $\mathrm{CsoS} 2 \mathrm{~A}$ in the assembly of $\alpha$ 41 carboxysomes. 


\section{Introduction}

45 Organisms that aerobically fix $\mathrm{CO}_{2}$ through the Calvin-Benson-Bassham Cycle face a

46 fundamental biochemical problem. The principal enzyme of this cycle, Ribulose-1,5-bisphosphate

47 Carboxylase / Oxygenase (RuBisCO), has both a low affinity for $\mathrm{CO}_{2}$ and promiscuous activity

48 for the competing $\mathrm{O}_{2}$ substrate [1]. Cyanobacteria and chemolithoautotrophic bacteria overcome

49 this problem using an integrated physiological strategy known as the Carbon Dioxide

50 Concentrating Mechanism (CCM) [2]. The CCM is a multicomponent mechanism - composed of

51 inorganic carbon transporters and an organelle-like structure called the carboxysome - that

52 function together to selectively deliver high concentrations of $\mathrm{CO}_{2}$ directly to $\mathrm{RuBisCO}$ in order

53 to facilitate carbon fixation.

54 The carboxysome is the centerpiece of the CCM. The carboxysome is an icosahedral 55 protein complex of $80-500 \mathrm{~nm}$ composed of thousands of protomers [3-5]. There are two

56 distinct evolutionary lineages of the carboxysome, $\alpha$ and $\beta$ forms, which differ in their protein

57 composition but share two major structural and functional features: i) a protein shell composed of

58 thousands of hexameric and 60 pentameric capsomers and ii) a lumen possessing numerous

59 copies of the cargo enzymes RuBisCO and carbonic anhydrase [6]. In concert with active

60 transport producing high levels of cytosolic bicarbonate, this structural arrangement leads to the

61 increased concentration of $\mathrm{CO}_{2}$ within the lumen, allowing RuBisCO to catalyze carbon fixation

62 with both high rate and specificity [7].

63 Recent work on the cyanobacterial $\beta$-carboxysome has identified a series of molecular 64 interactions that non-covalently target holo-RuBisCO (8 large subunits and 8 small subunits), the 65 major carboxysome component by molecular weight (MW), to the lumen of the shell. 66 Specifically, the protein CcmM contains three domains homologous to the small subunit of 67 RuBisCO and is capable of bridging multiple RuBisCO complexes together [8,9]. CcmM has 68 been shown to interact with a second factor, CcmN, which in turn interacts with the hexameric 69 shell protein CcmK2 [10]. Thus, $\mathrm{RuBisCO}$ is thought to form a nucleate-like aggregate in the 70 cytosol, facilitated by CcmM, and then matures into a complete carboxysome by the recruitment 71 of additional shells proteins by $\mathrm{CcmN}[11,12]$.

72 A significant variation between $\alpha$ and $\beta$ carboxysomes occurs at the level of the 73 intermediary factors bridging cargo and shell $[4,6,13]$. Specifically, the $\alpha$-carboxysome does not 
74 contain a $\mathrm{CcmM}$ or $\mathrm{CcmN}$ homolog and the assembly process is less characterized. Biochemical 75 and genetic analysis by Heinhorst and colleagues have implicated ten genes in the production of $76 \alpha$-carboxysomes in Halothiobacillus neapolitanus, and it has recently been shown that these 77 genes are sufficient for carboxysome expression in the non-native host E. coli [5,14-16]. Notably, 78 nine of these ten genes have an obvious function: six are unambiguously shell proteins and 79 encode for either bacterial microcompartment (BMC) domain-containing proteins ( $\operatorname{csoSIABCD}$; 80 pfam00936) or vertex-forming pentamers ( $\operatorname{csoS4AB}$; pfam03319) while three encode the cargo 81 enzymes carbonic anhydrase ( $c s o S 3)$ and RuBisCO ( $c b b L S)$ (Heinhorst 2006). The remaining 82 gene, $\operatorname{cso} 2$, has a less obvious functional role in the carboxysome. Early electron microscopy 83 (EM) studies localized the CsoS2 protein to the shell, but more recent two-hybrid, gel-shift, and 84 pull-down experiments suggest $\mathrm{CsoS} 2$ may also interact with a number of components including 85 the shell proteins and RuBisCO [17-19]. Moreover, deletion of csoS2 abolishes carboxysome 86 formation [18]. Thus, one possibility is that CsoS2 plays a role in carboxysome assembly and 87 may be functionally analogous to $\mathrm{CcmM}$ and $\mathrm{CcmN}$.

88 Much of the uncertainty in CsoS2 function lies in an unexpected observation: 89 carboxysomes from H. neapolitanus possess two different forms of CsoS2 [17]. The predicted 90 molecular weight of the $\operatorname{csos} 2$ gene product is $92 \mathrm{kDa}$ but purified carboxysomes possess a long 91 isoform, CsoS2B, and a short isoform, CsoS2A, with apparent MWs of $\sim 120$ and $\sim 70 \mathrm{kDa}$, 92 respectively, as analyzed by denaturing polyacrylamide gel electrophoresis (PAGE) analysis.

93 Previous work has shown that $\mathrm{CsoS} 2 \mathrm{~A}$ and $\mathrm{CsoS} 2 \mathrm{~B}$ share the same $\mathrm{N}$-terminus but may be post94 translationally modified via glycosylation [17], thus offering an explanation for the discrepancy 95 between predicted and observed MWs, although other evidence suggests the proteins may not be 96 modified [19,20].

97 Elucidating a clear functional role for $\mathrm{CsoS} 2$ requires understanding its specific 98 molecular interactions with other carboxysome components and, therefore, the precise definition 99 of primary amino-acid sequence structure for both CsoS2B and CsoS2A. Here, using a 100 heterologous E. coli expression system, we show that differential expression of the two isoforms 101 is encoded by ribosomal frameshifting cis elements present on the csoS2 mRNA that are capable 102 of encoding roughly 1:1 stoichiometric expression of CsoS2A and CsoS2B. CsoS2B is 869 amino 103 acids long and produced from full length expression of the 0 frame of the gene. Frameshifting 
104 specifically generates the CsoS2A protein possessing a C-terminus from the -1 frame and results 105 in a truncated protein 570 amino acids in length. Surprisingly, in carboxysome formation assays, 106 CsoS2B by itself leads to the formation of assemblies structurally similar to carboxysomes, while 107 CsoS2A alone is clearly incapable of forming carboxysomes. Thus, we have defined the sequence 108 of each CsoS2 isoform, a mechanism of their formation, and provide initial evidence towards 109 their functional importance.

110

111 


\section{Results}

114 Recent evidence suggests CsoS2 is crucial to the assembly of the carboxysome, but its

115 precise molecular function remains unclear due to the ambiguous relationship between CsoS2A 116 and CsoS2B [18]. To resolve this uncertainty, we sought to delineate the primary structure of 117 these two proteins and their relative functional importance.

118 The primary DNA sequence of $\operatorname{csoS} 2$ from $H$. neapolitanus encodes a 92.0 kDa protein.

119 Inspection of this amino-acid sequence reveals a protein with three regions, an N-terminal, 120 Middle, and C-terminal Region (NR, MR, and CR) (Fig. 1A). Previous bioinformatic analysis 121 suggested the NR possesses four homologous repeats with alpha helical propensity and that the

122 MR possesses six homologous repeats of roughly 50 amino acids in length each possessing three 123 canonical (V/I)(T/S)G motifs (R1-R6; Fig. 1A) [18]. Interestingly, inspection of the primary 124 sequence reveals the possibility of three additional repeats in the CR (Fig. S1A). These repeats 125 (R7-R9) possess extended interstitial amino acids compared to repeats in the MR. CsoS2 also 126 possesses an extremely conserved terminus, which we term the C-terminal Peptide (CTP) (Fig. 127 S1B). Finally, CsoS2 is also predicted to be highly intrinsically disordered, which has been 128 experimentally validated in solution scattering experiments [18].

129 In order to link this sequence with function, we set out to understand how one such gene 130 can generate two gene products. Heterologous expression of CsoS2 indicated that the mechanism

131 of differential expression used to generate CsoS2A and CsoS2B is conserved between the gamma 132 proteobacteria $E$. coli and $H$. neapolitanus. Carboxysomes purified from either $H$. neapolitanus 133 (HnCB) or E. coli (EcCB; see Bonacci et al. 2012) produce bands at the characteristic, anomalous 134 MWs of $120 \mathrm{kDa}$ and $70 \mathrm{kDa}$ and with roughly 1:1 stoichiometry (Fig. 1B). Likewise, expression 135 of CsoS2 protein bearing an N-terminal 6x polyhistidine tag (EcS2) also produced both the A and

136 B form, with a preference for CsoS2A possibly due to higher degradation of CsoS2B or selective 137 enrichment of CsoS2A during the purification procedure. Previous results indicate differential 138 expression may arise from post-translational processing [17] but attempts to verify glycosylation 139 or proteolytic activation were negative (Fig. S2).

140 Intrinsically disordered proteins often run at larger apparent MWs in denaturing PAGE, 141 offering a partial explanation for the observed anomalous masses [21]. We next verified that the 
142 EcS2 mass matched that of the theoretical gene product using Matrix-assisted Laser 143 Desorption/Ionization - Time of Flight (MALDI-TOF) Mass Spectrometry (MS). This revealed 144 two peaks with large $\mathrm{m} / \mathrm{z}$, corresponding to masses of $60.8 \mathrm{kDa}$ and $92.3 \mathrm{kDa}$ (Fig. 1C). The latter 145 is an identical (within $0.01 \%$ ) match to the predicted mass of full-length EcS2 and is expected to 146 be $\mathrm{CsoS} 2 \mathrm{~B}$. We therefore concluded that the $60.8 \mathrm{kDa}$ species corresponded to CsoS2A and 147 sought to identify, using this MW as a guide, the mechanism of CsoS2A expression.

148 The lack of post-translational processing led us to ask whether an alternative mechanism, 149 such as co-translational regulation via frameshifting, could explain the presence of CsoS2A. 150 Programmed ribosomal frameshifting (PRF) is a process whereby an mRNA encodes a specific 151 signal to shift the ribosome from the usual translated 0 frame to either the +1 or -1 frame [22].

152 This can result in the production of two or more different polypeptides from a single mRNA. 153 While pervasive in viruses, PRF is much less common in prokaryotes and eukaryotes. Only a 154 handful of proteins in E. coli, most notably the gamma subunit of DNA polymerase III, are 155 known to be generated by PRF [23].

156 Based on the MALDI-TOF data, we hypothesized that the terminal stop codon for 157 CsoS2A was likely to be in the latter half of the gene, due to its mass of $60.8 \mathrm{kDa}$. To probe for 158 PRF, we designed a construct, EcCsoS2-NonStop, possessing synonymous mutations ablating the 15922 stop codons in the -1 and +1 frames of the latter half of the gene. The construct also contained 160 stop codons in the -1 and +1 frames just downstream of the normal 0 frame stop. Strikingly, 161 expression of EcCsoS2-NonStop generated only a single band with MW similar to CsoS2B (Fig. 162 2A). Therefore, expression of a synthetic CsoS2 revealed that frameshifting is likely responsible 163 for the differential expression of the two isoforms.

164 Further MS analysis confirmed this hypothesis and identified the site of frameshifting. 165 CsoS2A and CsoS2B were isolated from PAGE bands of purified EcS2, trypsinized, and analyzed 166 using MS/MS. Masses from this experiment were matched to a theoretical MW library generated 167 from the CsoS2 sequence in order to identify peptides specific to each isoform. CsoS2A and 168 CsoS2B shared a large number of peptides in the first $2 / 3$ of the protein sequence, but there was a 169 dramatic reduction in CsoS2A peptides beyond amino acid 570 (Fig. 2B). The recent work of Cai 170 et al. noted a similar observation [18]. 
$171 \quad \mathrm{PRF}$ generates proteins possessing an $\mathrm{N}$-terminus from the 0 frame and a $\mathrm{C}$-terminus

172 from either the -1 and +1 frames. We therefore repeated the peptide identification analysis but 173 instead used a theoretical frameshifting-specific library, containing every possible frameshifted 174 polypeptide from the $\operatorname{csos} 2$ gene. Surprisingly, this analysis revealed a unique peptide 175 HAPKDVR - containing a fusion of amino acids from the 0 and -1 frames (Fig. 2C). The 176 theoretical mass of a CsoS2A protein ending in this sequence exactly matches the experimental 177 value of $60.8 \mathrm{kDa}$ from Fig. $1 \mathrm{C}$, thus providing additional confirmation.

178 The model system for -1 PRF is the gene dnaX from E. coli, which generates the gene 179 products tau and gamma of the DNA polymerase III complex [23,24]. A large body of 180 biochemical and biophysical data on this system have defined the signal for frameshifting: i) a 5' 181 ribosomal binding site upstream of the frameshifting site which stalls the ribosome, ii) a slippery 182 sequence of A-AAA-AAG where the ribosome changes frame, and iii) significant secondary 183 structure downstream of the slippery sequence to further impede ribosome translation (Fig. 3B) 184 [22]. These signals cause the ribosome slipping from a 0 frame codon back to the -1 frame codon. 185 This process is enabled by the degeneracy of the genetic code and allowance of mismatches at the 186 wobble codon position. As a consequence, Kornberg et al. have shown a sequence matching the 187 nucleotide motif X-XXY-YYZ has the potential to allow PRF [23].

188 Strikingly, the $\operatorname{csoS} 2$ gene contains similar cis elements to $\operatorname{dnaX}$ at the gene sequence 189 neighboring the sequence encoding the HAPKDVR peptide (Fig. 3B). Most importantly, csoS2 190 contains a putative slippery sequence, C-CCA-AAG, matching the X-XXY-YYZ motif, directly 191 where there is ambiguity between the 0 frame and -1 frame in the MS/MS data (arrow in Fig. 2C).

192 In addition, RNAstructure analysis [25] of the primary nucleotide sequence indicates a potential 193 stem-loop structure with significant stability $(25.7 \mathrm{kcal} / \mathrm{mol})$, downstream of the slippery 194 sequence. This loop contains the C-terminus of the putative HAPKDVR peptide and a stop codon 195 immediately following the arginine codon, suggesting this is the terminus of CsoS2A.

196 This model was tested by generating constructs deficient in PRF. First, csoS2 was recoded 197 to end with the HAPKDVR-stop sequence in the 0 frame (EcS2A). Expression of this construct 198 was equal in size to CsoS2A and confirmed its anomalous PAGE behavior (Fig. 3C). To test the 199 role of the slippery sequence and secondary structure elements, we next designed constructs with 200 a series of synonymous mutations to ablate either the slippery sequence (NoSlip) or the stem-loop 
201 (NoStem) and perturb PRF (orange and red circles in Fig. 3B). As with EcCsoS2-NonStop, these 202 constructs expressed a long peptide matching the MW of CsoS2B (Fig. 3C). Thus, both the 203 slippery sequence and the downstream secondary structure are required for -1 PRF.

204 To unambiguously assay the function of the PRF elements, we tested for frameshifting in 205 a completely synthetic context. A region of the $\operatorname{cso} 2$ gene containing the complete PRF elements and additional 15 base pairs downstream of the stem-loop was cloned between a green fluorescent 207 protein (GFP) and mCherry red fluorescent protein (RFP) reporter such that the 0 frame of the 208 PRF elements would yield a full length GFP-RFP fusion (Fig. S3). Expression of this construct 209 generated two fluorescent species - the expected full-length protein displaying both GFP and RFP

210 fluorescence, and a short form containing only GFP - thus confirming frameshifting (Fig. 3D).

211 Interestingly, the ratio of this construct favors the short form while the ratio of CsoS2A and 212 CsoS2B is roughly 1:1 from the carboxysome operon. Regardless, this result demonstrates the 213 PRF elements are robust to context, and are sufficient to recode the expression of a translating 214 message. Taken together, the above experiments suggest csoS2 undergoes a programmed 215 ribosomal frameshift to yield the different forms of CsoS2. More specifically, we conclude that 216 CsoS2A is a 570 amino acid protein whose terminal amino acids are encoded within the -1 frame 217 of the gene.

218 We next investigated the conservation of -1 PRF in CsoS2 homologs from other 219 organisms. The -1 PRF prediction server KnotInFrame [26], with additional criteria (see 220 Materials and Methods), was used to search for -1 PRF candidates among known CsoS2-bearing 221 species (Fig. S5). Intriguingly, csoS2 genes from 79 out of 162 species were predicted to undergo 222 frameshifting and the distribution of PRF across the phylogenetic tree appears to be somewhat 223 clustered, suggesting that this mechanism is widely used and conserved among the species with 224 closely related $\mathrm{CsoS} 2$ homologs. Although further experimental data are needed to validate each 225 prediction, we found that a number of them matched previously reported observations. For 226 instance, $H$. neapolitanus $\mathrm{CsoS} 2$ is predicted to frameshift at the location we experimentally 227 confirmed in this study and the predictions matched previous unexplained observations of 228 carboxysomes possessing either one or two CsoS2 isoforms in three different species ( $T$. 229 intermedia, T. crunogena and P. marinus str. MED4) [16,27]. Closer inspection of each 230 organism's characteristics, habitat, and CCM function may shed light on why -1 PRF exists in 
231 some bacteria but not in others.

232 Finally, we sought to determine the significance of each isoform for carboxysome 233 assembly. Mapping the location of PRF onto the primary sequence of CsoS2 shows that the site is 234 within Repeat 6 at the end of the MR. Thus, CsoS2A contains the NR and five complete repeats 235 within the MR (asterisk Fig. 1A), while CsoS2B contains the entire CR, including the highly 236 conserved CTP. We therefore hypothesized this variation in sequence could create a functional 237 difference between CsoS2A and CsoS2B.

238 The importance of these sequences was assayed using heterologous carboxysome 239 expression in E. coli. It has been shown previously that ten genes from two H. neapolitanus 240 operons can be refactored into a single isopropyl $\beta$-D-1-thiogalactopyranoside (IPTG)-inducible 241 operon and expressed in E. coli (EcCBs) [14]. EcCBs are generally well-formed and are 242 functional for $\mathrm{CO}_{2}$ fixation but, in contrast to native $\mathrm{HnCBs}$, are somewhat more heterogeneous 243 in morphology and can possess broken shells. Regardless, this provides a simple platform to 244 assay an in vivo reconstitution of carboxysome assembly as a function of CsoS2 sequence.

245 Expression of EcCBs in this assay revealed a critical functional difference between 246 CsoS2A and CsoS2B. The EcCB expression plasmid was modified to encode either CsoS2A, 247 CsoS2B (with ablated slippery sequence), or wild-type (WT) CsoS2, and carboxysomes were 248 expressed and analyzed for formation by sucrose gradient purification and EM. In an SDS-PAGE 249 analysis, WT and CsoS2B-containing carboxysomes produced similar gradient fractionation 250 patterns, but CsoS2A yielded no fractions characteristic of carboxysomes (Fig. 4A and S4). EM

251 of WT and CsoS2B showed the formation of carboxysome-like structures while the CsoS2A252 derived fraction lacked any significant assembly (Fig. 4B). Thus, CsoS2A, in the absence of 253 CsoS2B, is incapable of forming carboxysomes while CsoS2B by itself is sufficient for shell 254 assembly around the lumen and form carboxysome-like structures. 


\section{Discussion}

256 Frameshifting and the two forms of CsoS2. Since the initial biochemical

257 characterization of $\alpha$-carboxysomes, the anomalous behavior of CsoS2 expression has

258 confounded the determination of its molecular function. Here, we show that -1 programmed

259 ribosomal frameshifting is responsible for the genesis of the two forms of CsoS2.

$260-1$ PRF occurs when the ribosome is stalled during translation by 3' secondary structure 261 and, in certain cases, a 5' internal ribosomal binding site-like sequence. A slippery sequence of 262 the mRNA allows the tRNAs to shift backward to wobble base-pair with the codons in the -1 263 frame. Slipped ribosomes that continue translating in the -1 frame can then encounter a stop 264 codon and lead to early protein truncation (Fig. 3A). Inspection of the $\operatorname{csoS} 2$ nucleotide sequence 265 revealed that it possesses both a potential slippery sequence and a significant predicted 3' stem266 loop. A relatively purine-rich sequence (GGCCAA) is observed 14 nucleotides upstream of the 267 slippery sequence. However, since the sequence is significantly different from the consensus, it is 268 uncertain whether this sequence could act like a ribosome-binding site and contribute to PRF. 269 Removal of either the slippery sequence or stem-loop abolishes CsoS2A, suggesting that these 270 elements are necessary for frameshifting. In addition, PRF function is robust to sequence context,

271 as frameshifting can occur when the $\operatorname{csoS} 2$ gene is expressed by itself in E. coli or completely out 272 of context, as seen in the synthetic fluorescent reporter containing only the slippery sequence and 273 the stem-loop. Therefore, these elements are sufficient to explain the generation of two CsoS2 274 isoforms.

275 The efficiency of known -1 PRF elements can range from a few percent to as high as $80 \%$ 276 [22]. The CsoS2 PRF is 50\% and is therefore near the upper range of characterized examples 277 across all kingdoms of life. The dearth of model frameshifting systems in prokaryotes has made 278 the prediction of efficiency difficult, so CsoS2 therefore provides a novel example to better 279 inform this understanding. Clearly, extensive Watson-Crick base-pairing and high GC content in 280 the stem-loop (Fig. 3B) is important for efficiency, but the overall efficiency is likely to be 281 multivariate and context-dependent [28].

282 While we portray the -1 PRF of CsoS2 as entering the -1 frame when CCA and AAG 283 codons are at the $\mathrm{P}$ and $\mathrm{A}$ sites of the ribosome (Fig. 3A), respectively, a recent study has shown 284 that PRF can operate through multiple pathways simultaneously [29]. Specifically, the translation 
285 apparatus can enter the -1 frame from several codons along the slippery sequence and can also 286 slip by -4 or +2 nucleotides. Therefore, it is formally possible that CsoS $2 \mathrm{~A}$ in its native context 287 could be the product of not one, but several, translational pathways. Further mass spectrometry 288 studies, by probing for peptides from multiple frames, could elucidate the exact slip events that 289 occur during CsoS2 translation.

290 Finally, it is not known what advantages are conferred by using -1 PRF as opposed to 291 other strategies in generating isoforms. As -1 PRF is known to result in tight stoichiometric 292 control [30], it is possible that $\alpha$-carboxysome assembly benefits from having a fixed ratio 293 between CsoS2AB. Notably, in $\beta$-carboxysomes, CcmM has two isoforms, the short 35-kDa 294 (M35) and long 58-kDa (M58) proteins, the former generated via an internal ribosome entry site 295 (IRES) [8,31]. Despite different mechanisms, both -1 PRF and IRES involve mRNA secondary 296 structure, but direct comparison between the biological advantages of PRF and IRES is unclear. 297 One possibility is that size heterogeneity differences between $\alpha$ and $\beta$-carboxysomes [32] is 298 derived from the differences in function between $\mathrm{CsoS} 2$ and $\mathrm{CcmM} / \mathrm{CcmN}$ and the regulation of 299 their expression by PRF and IRES elements, respectively.

Frameshifting and the mechanism of carboxysome assembly. Several lines of evidence

302 hint at the possibility that CsoS2 may play a central role in organizing carboxysome assembly.

303 However, since it was previously not possible to individually express each form of CsoS2, the

304 functional difference between CsoS2A and CsoS2B has not been elucidated. Here, we use our

305 findings on PRF to specifically produce carboxysomes with either CsoS2A or CsoS2B in $E$. 306 coli. Surprisingly, we find that CsoS2B is necessary and, at least to the resolution of our 307 experiments, is sufficient for the assembly of carboxysomes similar to those possessing both 308 isoforms. Since CsoS2A is not required in this process, it is perplexing that this isoform is 309 produced in a significant amount in the native organism and that the frameshifting elements are 310 widely conserved across clades in the phylogeny of CsoS2. Future experiments, probing the 311 structure and function of carboxysome in a more fine-grained fashion, may reveal a role for the 312 CsoS2A isoform.

313 While we have clarified the molecular identity of each CsoS2 isoform, their exact 314 functions remain unclear. We speculate on their involvement in $\alpha$-carboxysome assembly by 
315 analogy to the CcmMN system. M35 has been shown to nucleate RuBisCO, and M58 can connect

316 the RuBisCO aggregate to the shell [8,31,33]. Along these lines, CsoS2A may interact with

$317 \mathrm{RuBisCO}$ in the core of the lumen, while CsoS2B binds RuBisCO close to the periphery and uses

318 the C-terminus to anchor them to the shell. Recent results place the C-terminus of CsoS2B either

319 bound to the shell or outside it, which supports this hypothesis. Strikingly, EM images and in

320 silico modeling suggested that the ratio of $\mathrm{RuBisCO}$ located immediately underneath the shell to

321 those distributed in the remainder of the lumen is close to $1: 1$, matching the ratio of CsoS2A to

322 CsoS2B in a carboxysome [34]. It is therefore possible that CsoS2A and CsoS2B, driven by the

323 latter's localization to the shell, reside in different zones within the carboxysome (Fig. 4C).

324 Further protein-protein interaction studies and higher resolution studies of $\alpha$-carboxysome 325 assembly are needed to test this hypothesis.

326 A major difference between CsoS2 and CcmM lies in the necessity of the isoforms. In $\beta$ -

327 carboxysomes, both CcmM isoforms are necessary for the assembly process [31]. RuBisCO 328 nucleation, mediated by M35, is required as the first step in the assembly [11]. On the other hand, 329 the formation of $\alpha$-carboxysomes likely proceeds without RuBisCO nucleation or at least in 330 concert with it, as $H$. neapolitanus carboxysomes can assemble in the absence of lumenal 331 RuBisCO [35,36]. The lack of requirement for CsoS2A, therefore, may indicate a different 332 assembly pathway.

333 Combining our findings above with previous work [18,20,32], we arrive at a potential 334 model describing assembly of the $\alpha$-carboxysome. Given previous results, CsoS2B is localized 335 near the shell and may facilitate RuBisCO's previously observed tight packing [34] in this region, 336 while $\mathrm{CsoS} 2 \mathrm{~A}$ primarily organizes $\mathrm{RuBisCO}$ in the remainder of the carboxysomal lumen (Fig. 337 4C). Several unresolved questions in this model warrant further experimental investigation. First, 338 the molecular recognition elements facilitating interaction between $\mathrm{CsoS} 2 \mathrm{AB}$ and the other 339 proteins remain unknown. M35, having three $\mathrm{RuBisCO}$-like repeats, can organize $\mathrm{RuBisCO}$ into 340 the previously observed aggregate. Repeats in CsoS2AB share no sequence similarity to 341 RuBisCO, so the outcome and quality of any such aggregation may be different. In addition, there 342 is a strong conservation of the last 30 amino acids of CsoS2B (i.e. the CTP), suggesting 343 functional importance, but a specific binding partner has not been identified. Second, while we 344 portray $\mathrm{CsoS} 2 \mathrm{~A}$ as residing with $\mathrm{RuBisCO}$ in the core of a carboxysome, we cannot rule out the 
345 possibility that some CsoS2A could exist in the subshell layer. Third, besides RuBisCO and shell

346 proteins, CsoS2 may also interact with carbonic anhydrase. Finally, due to a number of conserved

347 Cys residues, the two isoforms of CsoS2 may covalently interact with one another [18].

348 Evolutionary aspects of CsoS2. Although there is no direct evidence for a viral origin to

349 carboxysomes and other BMCs, their similarities are striking. Both types of particles are

350 polyhedral, proteinaceous, and self-assembling. CsoS2 also shares additional characteristics of

351 viral proteins: it is highly intrinsically disordered, and undergoes PRF. The latter is common

352 among viral proteins as viral constraints necessitate tight stoichiometric regulation and genetic

353 economy. For example, a functionally similar protein to CsoS2, VP4, is present in Foot-and-

354 Mouth-Disease virus. Like CsoS2, VP4 is an intrinsically disordered protein, binds to the interior

355 side of the capsid shell, and is necessary for assembly of the viral capsid [37,38]. It remains to be

356 seen whether these functional properties are, in fact, viral in origin or simply the result of the

357 functionality required to facilitate the structural self-assembly of protein capsids.

$358 \quad$ While $\alpha$ - and $\beta$-carboxysomes appear to be evolutionarily independent, they both employ

359 two protein isoforms and repeating units to mediate the assembly. Such a strategy might provide a

360 number of advantages. It has been reported that a multivalent protein can mediate the aggregation

361 of its partner in a switch-like manner [39]. Repeats in CsoS2 and CcmM may provide

362 multivalency for a similar mechanism. Moreover, in both classes of carboxysomes, the shell

363 interaction appears to be mediated by the long form of these proteins yet a shorter, multivalent

364 species is often present. These observations may hint at the nature and rules governing self-

365 assembly of these protein complexes and therefore serve as the basis for future experiments

366 interrogating the design principles of carboxysome assembly and function in the cytosolic milieu. 


\section{Materials and methods}

368 Preparation of plasmids

Plasmids were constructed using Golden-Gate Assembly and Phusion polymerase (New

370 England Biolabs). Primers were designed to contain BsaI restriction sites and Golden-Gate

371 recombination sites appropriate for the assembly of the desired construct. In those constructs with

372 an affinity tag, the sequence expressing the tag was also included in a primer. Expression vectors

373 were modified in our laboratory to be compatible with Golden-Gate cloning (Table S1).

374 The open reading frame for H. neapolitanus CsoS2 was amplified from the pHnCB10

375 plasmid containing nine genes of the $c$ so operon and $\operatorname{csoSID}$ (Bonacci 2012). For the construct

376 containing $\operatorname{csoS} 2$ without stop codons in alternative reading frames, gBlocks containing

377 synonymous mutations that remove stop codons in alternative frames, starting at the $1278^{\text {th }}$

378 nucleotide of $\operatorname{csoS} 2$, were ordered from Integrated DNA Technologies. The gBlocks were

379 amplified by PCR to generate BsaI restriction sites and then incorporated into the pET14-based

380 destination vector using Golden-Gate assembly.

381 For the construct expressing only $\mathrm{CsoS} 2 \mathrm{~B}$, a set of primers was designed to either 1)

382 change the slippery sequence from 5'-C-CCA-AAG-3' to 5'-A-CCT-AAG-3' or 2)

383 synonymously mutate several nucleotides in the downstream hairpin to reduce hairpin formation.

384 The sequence of the mutated hairpin region is shown in Fig. 3.

385 To make the frameshift reporter construct, PCR was employed to introduce the BsmBI

386 sites to the EGFP gene, the slippery sequence and stem-loop region in $c s o S 2$, and the mCherry

387 gene. A TEV protease cleavage site was also introduced between the stem-loop and mCherry.

388 These amplified products were assembled simultaneously into the pET14-based destination

389 vector via Golden-Gate assembly. The control construct, with the slippery sequence and stem-

390 loop replaced by 5'-GCGGCGGGCGGTGCAGGAGCT-3' (encoding Ala-Ala-Gly-Gly-Ala-Gly-

391 Ala linker), was prepared in a similar manner.

392

\section{Heterologous expression and purification of His-tagged $\boldsymbol{H}$. neapolitanus CsoS2}

Chemically competent E.coli BL21(AI) transformed with pBz13 were grown in 1 L LB

396175 RPM for 16 hours. Cells were subsequently harvested by centrifugation at 4000xg for 20 
397 minutes. The pellets were resuspended in Buffer A (20 mM Tris $300 \mathrm{mM} \mathrm{NaCl} \mathrm{pH} \mathrm{7.5)} \mathrm{with}$

398 cOmplete protease inhibitor (Roche), $0.1 \mathrm{mg} / \mathrm{ml}$ lysozyme (Sigma-Aldrich), and $25 \mathrm{U} / \mathrm{ul}$ 399 benzonase (EMD Millipore). Cell lysis was performed by passing the cell suspension through an

400 Avestin EmulsiFlex-C3 homogenizer three times. Crude lysate was centrifuged at 15,000xg for

40120 minutes to remove cell debris. Clarified lysate was incubated with Ni-NTA resin for 1 hour

402 and transferred to a gravity-flow column. The resin was washed with buffer A plus $30 \mathrm{mM}$

403 imidazole and $1 \mathrm{mM}$ phenylmethylsulfonyl fluoride. The protein was eluted with buffer A plus

$404300 \mathrm{mM}$ imidazole and $1 \mathrm{mM}$ phenylmethylsulfonyl fluoride. Eluted protein was passed through 405 10DG desalting column (Bio-Rad) to exchange into buffer B (50 mM Tris $150 \mathrm{mM} \mathrm{NaCl} \mathrm{pH} \mathrm{7.4)}$ 406 plus cOmplete EDTA-free protease inhibitor cocktail (Roche) and stored at $-80{ }^{\circ} \mathrm{C}$ until further 407 use.

\section{Expression and visualization of EGFP-mCherry frameshift reporter}

410 BL21(AI) transformed with pRJN-FS or pRJN-control were grown in LB media (10 ml)

411 At OD600 of 0.3-0.5, the cultures were induced with $0.1 \%$ arabinose and further grown at $22{ }^{\circ} \mathrm{C}$

412175 RPM for 16 hours. Cells were harvested by centrifugation at $4000 \times \mathrm{xg}$ for 20 minutes and 413 subsequently chemically lysed with SoluLyse-Tris (Genlantis). The lysate was clarified by 414 centrifuging at $15000 \mathrm{xg}$ for 20 minutes. The clarified lysate was mixed with SDS-PAGE sample 415 loading buffer. Without heating, gel samples were loaded on a 4-20\% TGX Criterion gel (Bio416 Rad). SDS-PAGE was performed at $150 \mathrm{~V}$ with Laemmli electrophoresis running buffer $(25 \mathrm{mM}$, 417 Tris $192 \mathrm{mM}$ Glycine, 1\% SDS). The gel was subsequently imaged with ChemiDoc imager (Bio418 Rad), using GFP and RFP filters to detect EGFP and mCherry signals, respectively.

\section{Western blotting}

421 Clarified lysates of BL21(AI) expressing the desired protein were mixed with SDS422 PAGE sample loading buffer and heated at $95{ }^{\circ} \mathrm{C}$ for 10 minutes. Gel samples were 423 electrophoresed on a 4-20\% TGX Criterion gel (Bio-Rad) at $200 \mathrm{~V}$ with Laemmli electrophoresis

424 running buffer and blotted to polyvinylidene fluoride membrane using a Trans-Blot Turbo 425 Transfer System (Bio-Rad). Proteins were subsequently probed with His-probe Antibody (H-3) 
426 HRP (Santa Cruz Biotechnology) and detected through chemiluminescence using SuperSignal

427 West Pico Chemiluminescent Substrate (Thermo Scientific)

\section{Purification of carboxysomes from Escherichia coli}

Tuner competent cells (Novagen) were transformed with a plasmid (pHnCB10LC) 431 expressing carboxysomes. Cells were grown in $1 \mathrm{~L}$ of Terrific Broth at $37^{\circ} \mathrm{C}$ to an OD600 of 0.3-

4320.5 at which point IPTG was added to a final concentration of $1 \mathrm{mM}$. The culture was induced for

43316 hours at $20{ }^{\circ} \mathrm{C}$ and harvested by centrifugation at 5000xg for 20 minutes.

434 The cell pellet was resuspended in $50 \mathrm{ml}$ Buffer TEMB (10 mM Tris $10 \mathrm{mM} \mathrm{MgCl} \mathrm{M}_{2} 1$ $435 \mathrm{mM}$ EDTA $20 \mathrm{mM} \mathrm{NaHCO} 3 \mathrm{pH}$ 8.4) with $1 \mathrm{mM}$ phenylmethylsulfonyl fluoride, $0.1 \mathrm{mg} / \mathrm{ml}$ 436 lysozyme (Sigma-Aldrich), and $25 \mathrm{U} / \mathrm{ul}$ benzonase (EMD Millipore). Cell lysis was performed by 437 passing the cell suspension through an Avestin EmulsiFlex-C3 homogenizer three times. The 438 crude cell extract was centrifuged at $12,000 \times$ g for 30 minutes to remove cell debris. The 439 supernatant was subsequently centrifuged at 40,000xg for 30 minutes to pellet the carboxysomes. 440 The pellet was then resuspended in $20 \mathrm{ml}$ of 33\% cellLytic B (Sigma-Aldrich) in TEMB buffer in 441 order to remove lipid contaminants and centrifuged again at $40,000 \mathrm{xg}$ for 30 minutes. The pellet 442 was resuspended in three $\mathrm{ml}$ TEMB and centrifuged at 3000xg. The supernatant was then applied 443 to $25 \mathrm{ml}$ sucrose gradient made from 10, 20, 30, 40, and 50\% sucrose. The gradient was 444 centrifuged at 105,000 xg for 30 minutes. Fractions of one $\mathrm{ml}$ were collected and analyzed for the 445 presence of carboxysomes via SDS-PAGE gel. The fractions that contained carboxysomes were 446 pooled and centrifuged at 105,000xg for 90 minutes to pellet the carboxysomes. Carboxysomes 447 were then resuspended in $1 \mathrm{ml}$ TEMB and stored at $4{ }^{\circ} \mathrm{C}$ 


\section{MALDI-TOF analysis}

The matrix was prepared by dissolving $15 \mathrm{mg}$ of sinapic acid (Sigma-Aldrich) in $1 \mathrm{ml}$ of

451 70:30 water:acetonitrile with 1\% TFA. Purified EcS2 was mixed with the matrix in 1:10 v/v ratio.

452 A volume of $2 \mu 1$ of protein-matrix mixture was spotted on a well in 384-well plate (DE1580TA,

453 Axima) and desiccated. Another $2 \mu 1$ of protein-matrix was spotted on the same well and

454 desiccated once more. MALDI-TOF was performed in Axima Performance (Shimadzu

455 Technology) with 180 units of power, accumulating 400 profiles.

457 Tandem Mass Spectrometry

458 Two forms of CsoS2 in purified EcS2 were separated via SDS-PAGE (4-20\% TGX gel,

459 Bio-Rad) and stained with Coomassie stain. Bands containing CsoS2A and CsoS2B were excised 460 and submitted to the UC Davis Proteomics Core Facility. Analysis was performed on a Q 461 Exactive ${ }^{\mathrm{TM}}$ Hybrid Quadrupole-Orbitrap. Acquired spectra were searched in Scaffold against the 462 wild-type CsoS2 sequence and the frameshifted sequence library generated as described in 463 Bioinformatic Methods below. The list of peptides was used to deduce the location of 464 translational frameshifting.

\section{Preparation of samples for negative stain electron microscopy}

$4675 \mu \mathrm{l}$ of a protein sample was added to a freshly glow-discharged carbon coated copper 468 grid (EMS) and allowed to incubate for two minutes. The grids were washed with deionized 469 water three times, before incubation with $5 \mu \mathrm{l}$ of $1 \%$ uranyl acetate for one minute. Excess liquid 470 was gently wicked off and the grid was allowed to air-dry for two minutes. Negative stain EM 471 was performed with a Tecnai 12 TEM (FEI) operated at $120 \mathrm{kV}$.

\section{Bioinformatic methods}

474 To construct a library of frameshifted sequence, we wrote a Python script to construct the 475 list of tryptic peptides that would result from digestion of frameshifted Csos2. The script is 476 available online at https://github.com/SavageLab/csos2. In brief, we generated all possible fusions

477 of the primary Csos 2 reading frame with the -1 and -2 reading frames, including only cases in 478 which the fusion event was between $30 \%$ and $80 \%$ of the way through the Csos 2 coding 
479 sequence. We then simulated a tryptic digest of these $\sim 900$ fusion proteins, generating a 480 comprehensive list of possible fusion peptides. This database was then used to match $\mathrm{m} / \mathrm{z}$ spectra 481 from gel bands corresponding to $\mathrm{Csos} 2 \mathrm{~A}$ and $\mathrm{Csos} 2 \mathrm{~B}$. The piling diagram was constructed from 482 peptide counts derived from comparing mass spectra of Csos2A and Csos2B gel bands to the 483 primary coding sequence of Csos2 (i.e. disregarding the potential for frameshifting). We counted 484 the number of times each amino acid was observed in a peptide matched against the primary 485 coding sequence. These counts are plotted in Figure 2 in the main text.

486 The secondary structure of mRNA was computed using the RNAstructure web server. 487 Nucleotide sequence starting at 50 bases 5' of the slippery sequence and ending at 50 bases 3' of 488 the slippery sequence was used as the input. The temperature parameter was set to $303.15 \mathrm{~K}$. To 489 calculate the stability of the stem-loop alone, the sequence corresponding to the predicted stem490 loop was entered without additional nucleotides

491 To construct a phylogenetic tree mapped with -1 PRF prediction (Fig. S5), species bearing $492 \operatorname{csoS} 2$ genes were curated from the Joint Genome Institute Integrated Microbial Genomes (JGI 493 IMG) database (https://img.jgi.doe.gov). FASTA sequences for the CsoS2-encoding mRNAs 494 were input into the KnotInFrame -1PRF prediction tool (https://bibiserv.techfak.uni-bielefeld.de). 495 Significant -1 PRF candidates as predicted by the KnotInFrame tool were further filtered by 496 setting a threshold such that the candidate slip sequence must be found in the last two thirds of the 497 gene and that the structured mRNA element immediately downstream of the 'XXXYYYZ' slip 498 sequence must have minimal free energy value $.025 \mathrm{kcal} / \mathrm{mol}$ more stable than the nested mRNA 499 structure. Species with candidate -1 PRF sequences meeting these parameters were marked in 500 green on the phylogeny of $\operatorname{csoS} 2$ nucleotide sequences.

\section{Acknowledgments}

503 We thank Avi Flamholz for bioinformatics-related consultation and the construction of the piling

504 diagram, Rayka Yokoo and Rachel Hood for critical reading of the manuscript, Brett Phinney 505 from the UC Davis Proteomics Core Facility for discussions about the MS/MS data, and Shannon 506 Yan for helpful discussions regarding PRF. This work was supported by the DOE Office of 507 Science Early Career Research Program (Grant number DE-SC0006394) through the Office of 508 Basic Energy Sciences and an Alfred P. Sloan Foundation Research Fellowship to D.F.S. 


\section{Author contributions}

511 T.C and D.F.S designed the research, analyzed the data, and wrote the manuscript; T.C performed

512 the experiments for Figure 1, 2, and S2; T.C. and R.J.N. performed the experiments for Figure 3;

513 T.C., K.E.K., C.F.N., P.K.T. and L.M.O. performed the experiments for Figure 4 and S4; R.J.N.

514 developed the algorithm for predicting -1 PRF in CsoS2 homologues and produced the

515 phylogenetic tree displayed in the supplementary information

516

517

518

519

520

521

522 


\section{Cited Literature}

525 [1] Tcherkez GGB, Farquhar GD, Andrews TJ. Despite slow catalysis and confused substrate specificity, all ribulose bisphosphate carboxylases may be nearly perfectly optimized. Proc Natl Acad Sci USA 2006;103:7246-51. doi:10.1073/pnas.0600605103.

529

530

[2] Badger MR, Price GD. $\mathrm{CO}_{2}$ concentrating mechanisms in cyanobacteria: molecular components, their diversity and evolution. J Exp Bot 2003;54:609-22. doi:10.1093/jxb/erg076.

[3] Shively JM, Ball F, Brown DH, Saunders RE. Functional organelles in prokaryotes: polyhedral inclusions (carboxysomes) of Thiobacillus neapolitanus. Science 1973;182:584-6.

[4] Yeates TO, Crowley CS, Tanaka S. Bacterial microcompartment organelles: protein shell structure and evolution. Annu Rev Biophys 2010;39:185-205. doi:10.1146/annurev.biophys.093008.131418.

[5] Heinhorst S, Cannon GC, Shively JM. Carboxysomes and carboxysome-like inclusions. In: Shively JM, editor. Complex Intracellular Structures in Prokaryotes, vol. II, Berlin: Microbiol Monogr; 2006, pp. 141-64.

[6] Cannon GC, Bradburne CE, Aldrich HC, Baker SH, Heinhorst S, Shively JM. Microcompartments in prokaryotes: carboxysomes and related polyhedra. Appl Environ Microbiol 2001;67:5351-61. doi:10.1128/AEM.67.12.5351-5361.2001.

[7] Dou Z, Heinhorst S, Williams EB, Murin CD, Shively JM, Cannon GC. $\mathrm{CO}_{2}$ fixation kinetics of Halothiobacillus neapolitanus mutant carboxysomes lacking carbonic anhydrase suggest the shell acts as a diffusional barrier for $\mathrm{CO}_{2}$. J Biol Chem 2008;283:10377-84. doi:10.1074/jbc.M709285200.

[8] Long BM, Badger MR, Whitney SM, Price GD. Analysis of carboxysomes from Synechococcus PCC7942 reveals multiple Rubisco complexes with carboxysomal proteins CcmM and CcaA. J Biol Chem 2007;282:29323-35. doi:10.1074/jbc.M703896200.

[9] Long BM, Rae BD, Badger MR, Price GD. Over-expression of the $\beta$-carboxysomal CcmM protein in Synechococcus PCC7942 reveals a tight co-regulation of carboxysomal carbonic anhydrase (CcaA) and M58 content. Photosynth Res 2011;109:33-45. doi:10.1007/s11120-011-9659-8.

[10] Kinney JN, Salmeen A, Cai F, Kerfeld CA. Elucidating Essential Role of Conserved Carboxysomal Protein CcmN Reveals Common Feature of Bacterial Microcompartment Assembly. J Biol Chem 2012;287:17729-36. doi:10.1074/jbc.M112.355305.

[11] Cameron JC, Wilson SC, Bernstein SL, Kerfeld CA. Biogenesis of a bacterial organelle: the carboxysome assembly pathway. Cell 2013;155:1131-40. doi:10.1016/j.cell.2013.10.044.

[12] Yokoo R, Hood RD, Savage DF. Live-cell imaging of cyanobacteria. Photosynth Res 2014;126:33-46. doi:10.1007/s11120-014-0049-x.

[13] Yeates TO, Kerfeld CA, Heinhorst S, Cannon GC, Shively JM. Protein-based 
604

605

606

607

608

609 organelles in bacteria: carboxysomes and related microcompartments. Nat Rev Micro 2008;6:681-91. doi:10.1038/nrmicro1913.

[14] Bonacci W, Teng PK, Afonso B, Niederholtmeyer H, Grob P, Silver PA, et al. Modularity of a carbon-fixing protein organelle. Proc Natl Acad Sci USA 2012;109:478-83. doi:10.1073/pnas.1108557109.

[15] Cai F, Heinhorst S, Shively JM, Cannon GC. Transcript analysis of the Halothiobacillus neapolitanus cso operon. Arch Microbiol 2007;189:141-50. doi:10.1007/s00203-007-0305-y.

[16] Roberts EW, Cai F, Kerfeld CA, Cannon GC, Heinhorst S. Isolation and characterization of the Prochlorococcus carboxysome reveal the presence of the novel shell protein CsoS1D. Journal of Bacteriology 2012;194:787-95. doi:10.1128/JB.06444-11.

[17] Baker SH, Lorbach SC, Rodriguez-Buey M, Williams DS, Aldrich HC, Shively JM. The correlation of the gene csoS2 of the carboxysome operon with two polypeptides of the carboxysome in Thiobacillus neapolitanus. Arch Microbiol 1999;172:233-9.

[18] Cai F, Dou Z, Bernstein S, Leverenz R, Williams E, Heinhorst S, et al. Advances in understanding carboxysome assembly in Prochlorococcus and Synechococcus implicate CsoS2 as a critical component. Life 2015;5:1141-71. doi:10.3390/life5021141.

[19] Gonzales AD, Light YK, Zhang Z, Iqbal T, Lane TW, Martino A. Proteomic analysis of the $\mathrm{CO}_{2}$-concentrating mechanism in the open-ocean cyanobacterium Synechococcus WH8102. Can J Bot 2005;83:735-45. doi:10.1139/b05-056.

[20] Espie GS, Kimber MS. Carboxysomes: cyanobacterial RuBisCO comes in small packages. Photosynth Res 2011;109:7-20. doi:10.1007/s11120-011-9656-y.

[21] Tompa P. Intrinsically unstructured proteins. Trends Biochem Sci 2002;27:527-33.

[22] Caliskan N, Peske F, Rodnina MV. Changed in translation: mRNA recoding by -1 programmed ribosomal frameshifting. Trends Biochem Sci 2015;40:265-74. doi:10.1016/j.tibs.2015.03.006.

[23] Tsuchihashi Z, Kornberg A. Translational frameshifting generates the gamma subunit of DNA polymerase III holoenzyme. Proc Natl Acad Sci 1990;87:2516-20.

[24] Flower AM, McHenry CS. The gamma subunit of DNA polymerase III holoenzyme of Escherichia coli is produced by ribosomal frameshifting. Proc Natl Acad Sci 1990;87:3713-7.

[25] Reuter JS, Mathews DH. RNAstructure: software for RNA secondary structure prediction and analysis. BMC Bioinformatics 2010;11:129. doi:10.1186/14712105-11-129.

[26] Theis C, Reeder J, Giegerich R. KnotInFrame: prediction of -1 ribosomal frameshift events. Nucleic Acids Res 2008;36:6013-20. doi:10.1093/nar/gkn578.

[27] Dou Z, University of Southern Mississippi. Department of Chemistry and Biochemistry. Functional characterization and assembly studies of carboxysomes in Halothiobacillus Neapolitanus. 2009.

[28] Mouzakis KD, Lang AL, Vander Meulen KA, Easterday PD, Butcher SE. HIV-1 frameshift efficiency is primarily determined by the stability of base pairs positioned at the mRNA entrance channel of the ribosome. Nucleic Acids Res 
2013;41:1901-13. doi:10.1093/nar/gks1254.

[29] Yan S, Wen J-D, Bustamante C, Tinoco I. Ribosome excursions during mRNA

[30] Xu J, Hendrix RW, Duda RL. Chaperone-protein interactions that mediate assembly of the bacteriophage lambda tail to the correct length. J Mol Biol 2014;426:1004- 


\section{Figure legends}

647 Fig. 1. CsoS2 is differentially expressed as two proteins without any post-translational 648 modifications. (a) A schematic representation of different regions in CsoS2, including N-terminal 649 Region (NR), Middle Region (MR), C-terminal Region (CR), and a conserved C-terminal Peptide 650 (CTP). NR contains 4 short repeats (yellow). MR has 6 longer repeats (R1-R6). CR has 3 repeats 651 (R7-R9). The repeats are divided by interstitial amino acids (grey), which do not align with any of 652 the repeats. The short isoform, CsoS2A, ends within R6 (asterisk). (b) SDS-PAGE gel of 653 carboxysomes purified from $H$. neapolitanus $(\mathrm{HnCB})$ and from recombinant expression in $E$. coli 654 (EcCB), and CsoS2 recombinantly expressed in E. coli (EcS2). The gel is annotated with the 655 identity of each protein band and molecular weight markers. (c) MALDI-TOF spectrum obtained 656 from purified EcS2.

658 Fig. 2. The csoS2 gene encodes a programmed ribosomal frameshift event. (a) Western blot of 659 CsoS2 (EcS2) and CsoS2-NonStop (NonStop) expressed in E. coli. (b) Piling diagram prepared 660 from the list of peptides discovered by MS/MS. Scale is normalized to maximum count for each 661 sample. CsoS2A is colored translucent green; CsoS2B in blue. (c) Diagram showing csoS2 662 codons in the 0 and -1 frames where the -1 PRF occurs. Codons in the -1 frame are underlined. 663 Amino acids in the 0 and -1 frames are written above and below the codons, respectively. The 664 arrow denotes the last amino acid shared between CsoS2A and B. The diagram is mapped back to 665 the corresponding region in the piling diagram (marked by dashed lines)

667 Fig. 3. Both the slippery sequence and the secondary structure in $\operatorname{csos} 2 \mathrm{mRNA}$ are necessary for 668 frameshifting. (a) A predicted stem-loop in the $\operatorname{csoS} 2$ mRNA causes the ribosome to stall during 669 translation. The ribosome may continue translating in the 0 frame, resulting in CsoS2B. 670 Alternatively, the slippery sequence allows the ribosome to slip backward to wobble base-pair 671 and continue translating in the -1 frame to generate $\operatorname{CsoS} 2 \mathrm{~A}$. Codons in the original frame are 672 marked by horizontal lines underneath. (b) Predicted secondary structure of the slip sequence and 673 downstream stem-loop (bp 1694-1746). Point mutations were made either in the slippery 674 sequence (orange) or the stem-loop (red). Bases at the end of the arrows outside of the structure 675 are those in the mutated products. Pink and blue bars indicate the slippery sequence and the -1 
676 frame stop codon, respectively. (c) Western blot of recombinant wild-type CsoS2 (EcS2), 677 CsoS2A (EcS2A), CsoS2 with an ablated slippery sequence (NoSlip), and CsoS2 with an ablated 678 stem-loop (NoStem). D) GFP fluorescence detected on the SDS-PAGE gel of synthetic EGFP679 RFP fluorescent reporters containing a linker (control) or PRF elements (PRF).

681 Fig. 4. Heterologous expression of carboxysomes without either CsoS2A or CsoS2B. (a) SDS682 PAGE of purified carboxysomes containing both CsoS2 isoforms (WT), CsoS2B only (S2B), and 683 CsoS2A only (S2A). Each sample was prepared from fraction 16 of the sucrose gradient (Fig. 684 S4). (b) Electron micrographs of the carboxysome samples used for SDS-PAGE gel. (Scale bar: $685100 \mathrm{~nm}$ ). (c) Model of possible differential functions of CsoS2 isoforms, in which CsoS2A 686 organizes lumenal RuBisCO while CsoS2B bridges the cargo to the shell. C-terminus of CsoS2B 687 is colored as blue. Areas with unresolved questions are circled with orange dashed lines: 1) the 688 location of CsoS2A; 2) the possibility and the nature of an interaction between CsoS2A and $689 \mathrm{CsoS2B}$; 3) the exact regions of CsoS2A or B that interact with RuBisCO; and 4) the location of 690 the C-terminus of CsoS2B.

691 
Chaijarasphong et al. 2015

Figure 1

(a) H. neapolitanus CsoS2
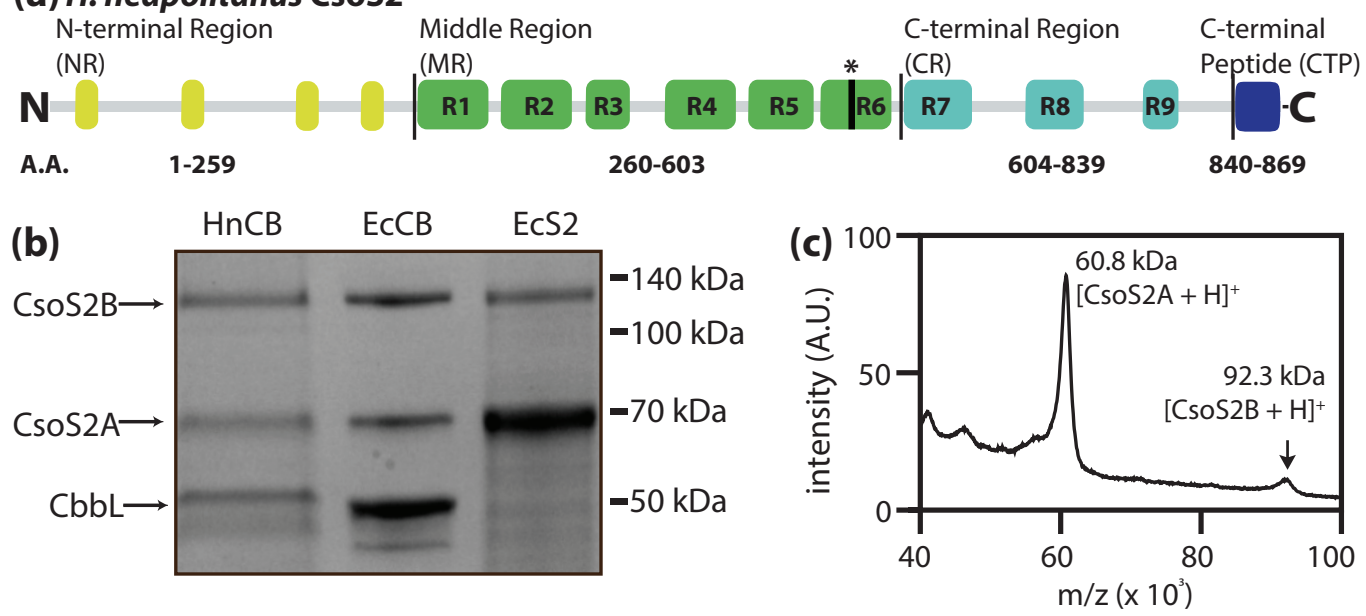
Figure 2
Chaijarasphong et al. 2015
Figure 2

Figure 2
Chaijarasphong
Figure 2

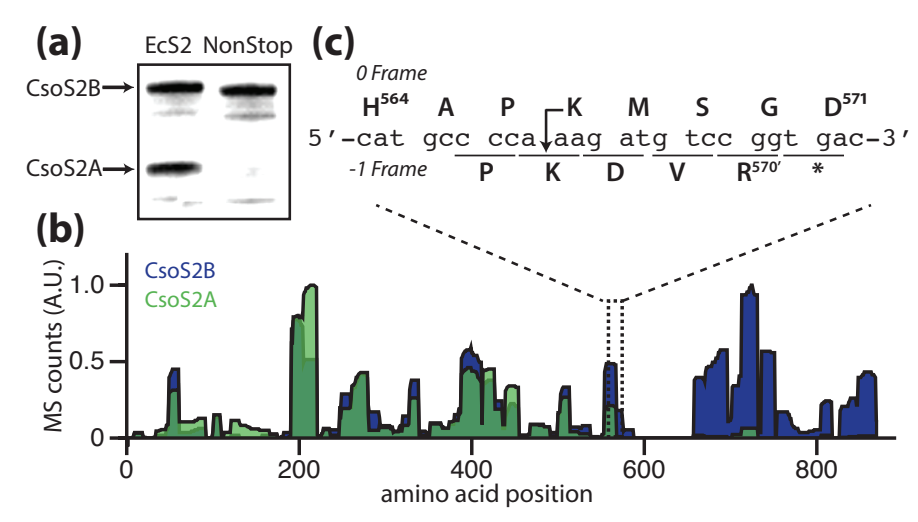


Figure 3

Chaijarasphong et al. 2015

Figure 3
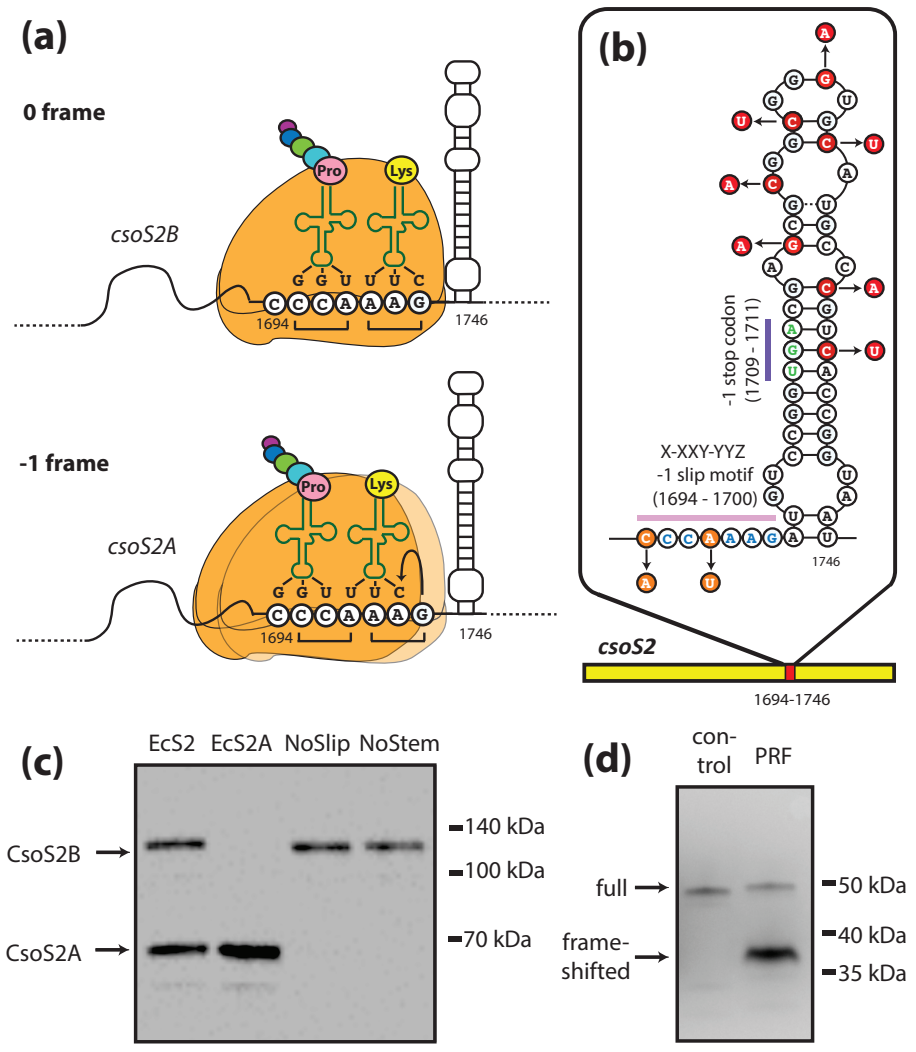
Figure 4

Chaijarasphong et al. 2015

Figure 4
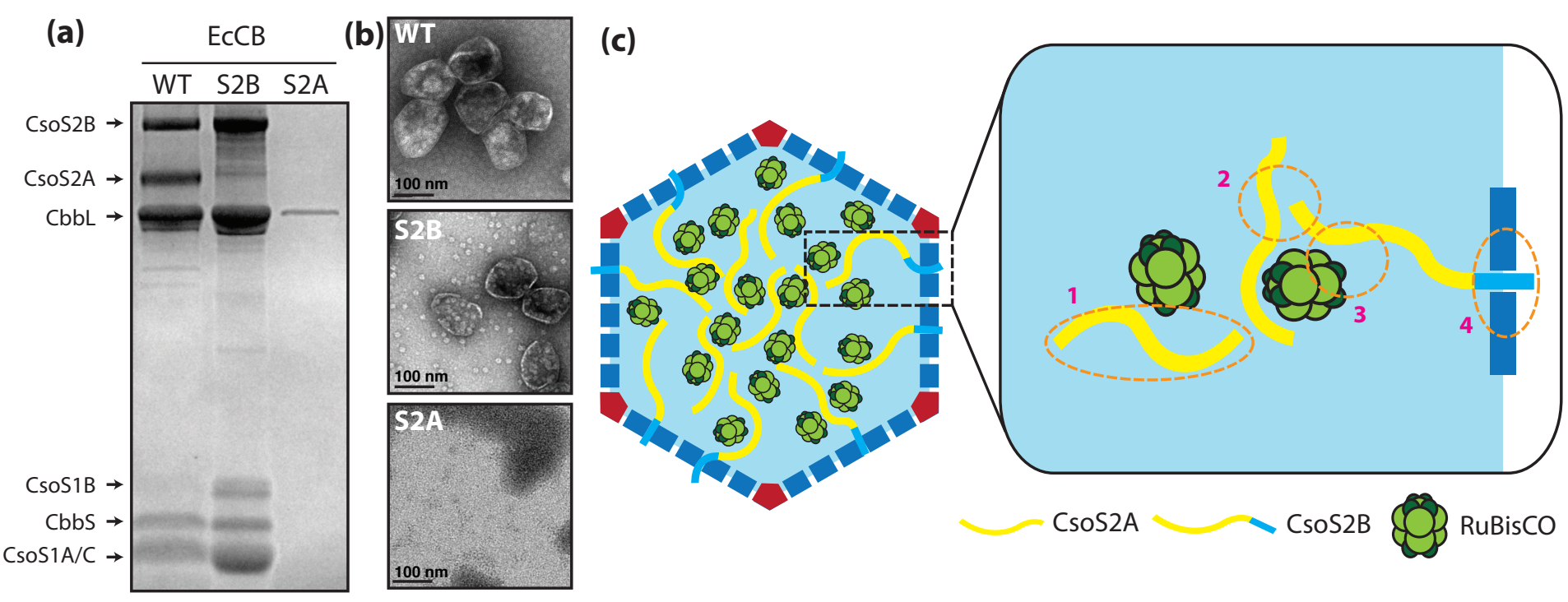